\title{
Qualidade de vida e transplante de células-tronco hematopoéticas alogênico: um estudo longitudinal'
}

\author{
Quality of life following allogeneic, hematopoietic \\ stem cell transplantation: a longitudinal study
}

\author{
Aline Bicalho MATIAS ${ }^{2}$ \\ Érika Arantes de OLIVEIRA-CARDOSO \\ Ana Paula MASTROPIETRO \\ Júlio César VOLTARELLI ${ }^{3}$ \\ Manoel Antônio dos SANTOS ${ }^{4}$
}

\begin{abstract}
Resumo
Este estudo teve como objetivo avaliar a qualidade de vida de pacientes submetidos ao transplante de células-tronco hematopoéticas alogênico, comparando as fases pré-transplante de células-tronco hematopoéticas, o momento do isolamento protetor na Enfermaria e o pós-transplante de células-tronco hematopoéticas imediato. A amostra foi composta por sete pacientes (quatro homens e três mulheres) submetidos ao transplante de células-tronco hematopoéticas no primeiro semestre de 2008. Na coleta de dados foi utilizado o Questionário Genérico de Avaliação de Qualidade de Vida (SF-36), aplicado individualmente em situação face a face. A comparação das médias obtidas nas fases pré-transplante de células-tronco hematopoéticas e Enfermaria evidenciou diferença estatisticamente significante nos domínios Capacidade Funcional $(p=0,022)$ e Dor ( $p=0,036)$. Comparando-se as etapas da Enfermaria e do pós-transplante de células-tronco hematopoéticas, evidenciou-se diferença significativa no Estado Geral de Saúde $(p=0,036)$. Não foram encontradas diferenças estatisticamente significantes entre as variáveis no pré e pós-transplante de células-tronco hematopoéticas. Desse modo, comparando-se as três etapas do transplante foi possível verificar que houve depreciação da qualidade de vida durante o isolamento protetor na Enfermaria, seguida de recuperação dos aspectos mensurados.
\end{abstract}

Unitermos: Células-tronco. Qualidade de vida. Saúde mental. Transplante de medula óssea.

\footnotetext{
Abstract

This research aimed to assess the quality of life of patients undergoing allogeneic, hematopoietic stem cell transplantation, comparing the pre-hematopoietic stem cell transplantation phase with the phase of protective isolation in the ward and the immediate post-hematopoietic stem cell transplantation. The sample comprised seven patients (four men and three women) who were submitted to

$\boldsymbol{\nabla \nabla \nabla \nabla}$

1 Artigo elaborado a partir do projeto intitulado "Qualidade de vida e aspectos psicológicos de pacientes que aguardam o transplante de medula óssea: um estudo longitudinal". Universidade de São Paulo, 2010. Apoio: Fundação de Apoio à Pesquisa do Estado de São Paulo, processo n 2007/58648-5.

2 Universidade de São Paulo, Membro do Núcleo de Ensino e Pesquisa em Psicologia da Saúde. Ribeirão Preto, SP, Brasil.

3 Universidade de São Paulo, Faculdade de Medicina de Ribeirão Preto, Hospital das Clínicas, Unidade de Transplante de Medula Óssea. Ribeirão Preto, SP, Brasil.

4 Universidade de São Paulo, Faculdade de Filosofia, Ciências e Letras de Ribeirão Preto, Departamento de Psicologia e Educação. Av. Bandeirantes, 3900 , 14040-901, Ribeirão Preto, SP, Brasil. Correspondência para/Correspondence to: M.A. SANTOS. E-mail: <masantos@ffclrp.usp.br>.
} 
this procedure in the first half of 2008. For the data collection, the Medical Outcomes Study 36-Item Short-Form Health Survey (SF-36) was used and individually applied in face-to-face situations. The comparison between mean data collected in the pre-hematopoietic stem cell transplantation phase and in the ward revealed a statistically significant difference in Functional Capacity $(p=0.022)$ and Pain ( $p=0.036)$. When comparing the ward and post-hematopoietic stem cell transplantation phases, a significant difference in General Health ( $p=0.036)$ was clearly shown. There was no statistically significant difference between the pre-and post-hematopoietic stem cell transplantation variables. Thus, when comparing the three transplantation phases, it was found that the quality of life diminished during protective confinement in the ward, followed by the recovery of the evaluated aspects.

Uniterms: Stem cells. Quality of live. Mental health. Bone marrow transplantation.

OTransplante de Células-Tronco Hematopoéticas (TCTH) constitui um recurso terapêutico utilizado para uma série de doenças malignas e para várias doenças congênitas e adquiridas de origem hematopoética, genética ou imunológica (Mastropietro, Oliveira-Cardoso, Simões, Voltarelli \& Santos, 2010; Mendes, Bittencourt \& Dusse, 2010). A medula óssea é um tecido líquido, também conhecido como tutano, que ocupa o interior dos ossos. Nesse tecido são formadas as hemácias, leucócitos e plaquetas, principais componentes do sangue. Quando ocorre o comprometimento da medula óssea, por doenças hematológicas ou neoplasias, e os tratamentos convencionais não são eficientes ou não oferecem possibilidades de melhora ao paciente, o TCTH é indicado como alternativa terapêutica (Thomas, 2000).

OTCTH ou Transplante de Medula Óssea (TMO), como ainda é mais conhecido - consiste na substituição da medula doente por células normais de medula óssea. Assim, a nova medula infundida passará a assumir a produção das células sanguíneas (Thomas, 2000). O procedimento é altamente complexo e pode ser dividido em três fases distintas: pré-TCTH, TCTH propriamente dito e pós-TCTH (Oliveira, Santos, Mastropietro \& Voltarelli, 2009; Riul \& Aguillar, 1997).

O pré-TCTH é marcado pelo momento pré-admissional, quando são realizadas avaliações pela equipe multidisciplinar. Em seguida, é feita a admissão hospitalar do paciente. A internação visa à realização do regime de condicionamento e imunossupressão, provocada por doses elevadas de quimioterapia. A aplasia medular exige que o paciente seja mantido em rigoroso regime de isolamento protetor. O TCTH propriamente dito é uma fase caracterizada pela coleta da medula óssea do doador (no caso do transplante alogênico), processamento e infusão de medula no paciente, e o enxertamento da medula óssea, a denominada "pega" da medula. Já o pós-TCTH é uma etapa 188 caracterizada pela alta hospitalar, quando o enxer- tamento é considerado bem-sucedido e não se observam complicações decorrentes do transplante (Guimarães, Oliveira-Cardoso, Mastropietro, Voltarelli \& Santos, 2010; Oliveira-Cardoso, Santos, Mastropietro \& Voltarelli, 2009b).

Embora não se trate de um processo cirúrgico, mas de uma infusão indolor, semelhante à transfusão sanguínea, oTCTH acarreta sérios riscos para o paciente, desde a perda da integridade física até o risco de morte. Por esse motivo, é considerado um tratamento que pode curar e, ao mesmo tempo, que pode levar ao óbito (Álvares, Bach \& Campos, 2003).

Assim, os primeiros conflitos psicológicos enfrentados pelos pacientes antecedem o transplante em si. Muitos dos candidatos ao TCTH têm como doença de base neoplasias hematológicas. A descoberta do câncer hematológico, ou de uma outra doença hematológica grave, é um momento especialmente difícil para os pacientes. Além do impacto causado pelo diagnóstico, muitas vezes incrementado pela não resolubilidade dos tratamentos convencionais, é comum que os pacientes vivenciem o conflito em relação à tomada de decisão pela realização ou não do transplante (Cooper \& Powell, 1998; Oliveira \& Santos, 2009; Oliveira-Cardoso et al., 2009b), uma vez que o TCTH surge como uma real possibilidade curativa, porém, simultaneamente, induz medo e acentua incertezas, pois aumenta o risco de o paciente ter sua sobrevida abreviada em decorrência de complicações do próprio procedimento. Essa ambiguidade incontornável desperta sentimentos ambivalentes de medo e esperança (Álvares et al., 2003; Guimarães, Santos \& Oliveira, 2008).

É comum, ainda, que o paciente se preocupe com sua condição física, enfrente complicações na fase pós-TCTH, interrupções de projetos e descontinuidade de sua vida social devido ao longo período de internação, quimioterapia agressiva, regime de isolamento 
protetor durante o período da aplasia medular, sem contar os inúmeros efeitos adversos do tratamento a longo prazo, como infertilidade, disfunção hormonal e, em alguns casos, recidiva da doença de base. Além disso, a pessoa submetida ao transplante também pode amargar dificuldades financeiras durante o tratamento (Lesko, 1990; Matsubara, Carvalho, Canini \& Sawada, 2007). Tais estressores podem interferir de modo negativo na qualidade de vida dos pacientes (Oliveira \& Santos, 2009; Oliveira-Cardoso et al., 2009b).

Nos últimos anos, a qualidade de vida tem sido estudada em diferentes contextos de saúde e doença, uma vez que a enfermidade não afeta somente a dimensão física, mas também repercute sobre o estado psicológico do indivíduo, seu nível de independência e suas relações sociais, fatores que extrapolam o objeto da saúde da medicina tradicional (Orley \& Kuyken, 1994). Qualidade de Vida Relacionada à Saúde (QVRS) refere-se a um conceito multidimensional que abrange a percepção do indivíduo a respeito de sua posição na vida, dentro de um contexto cultural e de um sistema de valores nos quais ele está inserido, relacionados aos seus objetivos, expectativas, padrões e preocupações (Fleck et al., 1999). Nesse contexto, recentemente a qualidade de vida de pacientes submetidos ao TCTH tem recebido maior atenção, em decorrência do aprimoramento da técnica, que tem elevado o índice de sobrevida dos pacientes, permitindo evolução mais favorável dos quadros.

Estudo realizado com pacientes candidatos ao TCTH mostrou que cerca de metade dos pacientes no momento pré-TCTH enfrentam níveis significativos de sofrimento psíquico (Trask et al., 2002). São diversos os fatores que podem interferir de maneira negativa na QVRS de pacientes submetidos ao TCTH, dentre eles: disfunções sexuais, desajustamento psicossocial e vocacional, estresse psicológico e manifestações psicopatológicas decorrentes dos efeitos adversos da própria terapêutica (Heinonen, Volin, Zevon, Uutela, Barrick \& Ruutu, 2005; Molassiotis \& Morris, 1999; Oliveira \& Santos, 2009).

Em estudo recente, realizado por Gregurek, Brajkovic, Kalenic, Bras e Persic-Brida (2009), observou-se correlação estatisticamente significativa entre ansiedade e qualidade de vida. Assim, com o aumento dos sintomas de ansiedade, houve declínio do nível de qualidade de vida. Contribuindo, ainda, para o rebaixamento da qualidade de vida dos pacientes durante a realização do transplante, Heinonen et al. (2005) chamam a atenção para o fato de o TCTH ser reconhecido como uma das estratégias terapêuticas mais estressantes no tratamento de certos tipos de câncer. Os autores apontam alguns aspectos que contribuem para a instalação de quadros de estresse, tais como: mudanças drásticas de vida, longa duração do tratamento, períodos de hospitalização e confinamento, efeitos colaterais dos procedimentos utilizados, medo da morte e falta de informações.

Fife, Huster, Cornetta, Kennedy, Akard e Broun (2000) realizaram um estudo longitudinal com 101 participantes submetidos ao TCTH, avaliando-os por meio de uma escala autoaplicável, nos momentos: antes da internação, pouco antes da infusão da medula óssea, sete dias e 14 dias após o transplante, e depois de um mês, três meses e 12 meses após a hospitalização. Foi encontrada maior depreciação do sofrimento emocional após a admissão hospitalar e antes da infusão da nova medula. Uma semana após o transplante foi observada uma diminuição dos sintomas de ansiedade e depressão. Além disso, confirmou-se que os três meses e um ano após o TCTH foram os períodos de menor sofrimento emocional.

Acompanhando essas evidências, McQuellon et al. (1998) publicaram um estudo prospectivo no qual avaliaram 74 pacientes do período pré-TCTH até um ano pós-TCTH. Os autores concluíram que, durante a internação, os pacientes apresentaram os piores índices gerais de qualidade de vida, havendo uma melhora progressiva em 100 dias e um ano, na medida em que recuperavam o convívio familiar e comunitário.

No pós-TCTH, principalmente no período imediato, os pacientes sofrem mais intensamente com as consequências do tratamento. Observa-se prejuízo no funcionamento físico, saúde geral, vitalidade e funcionamento social e emocional. O rebaixamento da qualidade de vida no pós-TCTH pode estar associado com ao longo tempo de internação, fraqueza física e prevalência de sintomas físicos e de ansiedade, depressão e estresse (Hann, Jacobsen, Martinho, Kronish, Azzarello \& Fields, 1997; Peres \& Santos, 2009; Trask et al., 2002).

Goetzmann et al. (2007) apontam a importância do acompanhamento psicossocial durante a evolução 
do tratamento, principalmente para pacientes com alta vulnerabilidade na etapa pré-TCTH, a fim de favorecer melhor adaptação e ajustamento às etapas pós-TCTH. Apesar das dificuldades mencionadas, muitos sobreviventes ao transplante alcançam uma boa condição de saúde, bons níveis de capacidade funcional e baixos níveis de angústia e sofrimento psicológico. São capazes de se adaptarem socialmente e de reassumirem papéis ocupacionais (Haberman, Bush, Young \& Sullivan, 1993).

No Brasil, ainda são escassas as investigações científicas sobre os aspectos psicológicos experimentados pelos pacientes submetidos ao TCTH, apesar do índice cada vez mais elevado de sobrevida dos portadores de doenças hematológicas e da angústia que vivenciam para decidirem pela realização ou não do procedimento, que implica riscos significativos, proporcionais ao seu potencial benefício (Álvares et al., 2003; Oliveira \& Santos, 2009).

Assim, o objetivo do presente estudo foi avaliar comparativamente a qualidade de vida de pacientes submetidos ao TCTH durante as três primeiras fases do transplante: pré-TCTH, Enfermaria (TCTH propriamente dito) e pós-TCTH imediato.

\section{Método}

\section{Participantes}

A amostra foi composta por sete pacientes, quatro homens e três mulheres, com idades variando entre 37 e 52 anos. Todos os homens participantes do estudo eram casados ou viviam em união estável e, entre as mulheres, apenas uma era solteira. Em relação ao status profissional, os homens exerciam atividades como contador, vendedor, pedreiro e policial militar, e as mulheres eram donas de casa e empregada doméstica. Os participantes apresentavam diferentes doenças de base: Leucemia Linfóide Aguda, Leucemia Mielóide Aguda, Linfoma Não-Hodgkin, Mieloma Múltiplo, Síndrome Mielodisplásica, Anemia de Fanconi, sendo que esta última condição havia acometido dois participantes. Todos tinham indicação para o TCTH alogênico, procedimento no qual a fonte de células-tronco hematopoéticas é um doador geneticamente similar.

Para seleção dos participantes foram adotados 190 os seguintes critérios de inclusão: pacientes maiores de
18 anos, de ambos os sexos, com indicação para o transplante alogênico, que aceitaram participar da pesquisa. Foram excluídos os pacientes com evidência de comprometimento psiquiátrico, intelectual e/ou debilidade orgânica, ou qualquer outra condição que pudesse prejudicar a compreensão ou o desempenho no instrumento aplicado.

\section{Instrumento}

Foi utilizado o Questionário Genérico de Avaliação de Qualidade de Vida (SF-36), traduzido e adaptado para a língua portuguesa por Ciconelli (1997), a partir do Medical Outcomes Study 36 Item Short-Form Health Survey.Trata-se de um instrumento de avaliação genérica de saúde, multidimensional, elaborado originalmente na língua inglesa, de fácil administração e compreensão. Esse instrumento tem sido largamente aplicado nos estudos que investigam oTCTH, tanto em contexto nacional como internacional. (Guimarães et al., 2008).

O SF-36 tem por objetivo mensurar as necessidades humanas básicas, o bem-estar emocional e funcional (Ware \& Sherboune, 1992). É constituído por 36 questões, que abrangem componentes físicos e mentais da QVRS. Dentre os primeiros, destacam-se os aspectos (ou domínios): Capacidade Funcional, que avalia as limitações relacionadas às atividades físicas; Aspectos Físicos, que afere o quanto as limitações físicas dificultam a realização de atividades diárias; Dor, que mensura a interferência da dor nas atividades da vida diária; Estado Geral de Saúde, que avalia a percepção do indivíduo sobre sua saúde e motivação pessoal. Dentre os componentes mentais, destacam-se: Aspectos Sociais, que avalia a frequência da interferência nas atividades sociais devido a problemas físicos ou emocionais; Vitalidade, que examina os sentimentos de cansaço e exaustão; Aspectos Emocionais, que mensura as limitações para realizar atividades devido à interferência de problemas emocionais; Saúde Mental, que avalia a percepção de sintomas de ansiedade, depressão, alteração do comportamento e descontrole emocional.

\section{Procedimentos}

Após aprovação do projeto pelo Comitê de Ética em Pesquisa da instituição hospitalar, a coleta de dados 
foi realizada nas dependências da Unidade de Transplante de Medula Óssea (UTMO) da Faculdade de Medicina de Ribeirão Preto da Universidade de São Paulo (FMRP-USP). Os pacientes que atendiam aos critérios de inclusão e concordaram em participar do estudo assinaram o Termo de Consentimento Livre e Esclarecido e responderam ao SF-36 em três momentos distintos: no momento da internação para início do procedimento, cerca de oito dias após a infusão da nova medula óssea e, aproximadamente, 35 dias após a alta hospitalar.

Para análise dos dados foram seguidas as instruções de Ciconelli (1997). Após a aplicação do instrumento, foi atribuído um escore para cada questão, posteriormente transformado em uma escala de 0-100, sendo cada dimensão analisada separadamente. Em decorrência do próprio construto de qualidade de vida, não existe um valor geral estimado pelo instrumento.

Para a análise estatística foi utilizado o teste não-paramétrico de Wilcoxon para amostras dependentes, escolhido por se tratar de uma amostra reduzida e com variáveis pareadas, considerando-se um valor de $p \leq 0,05$ para estimar o nível de significância.

\section{Resultados}

Como pode ser visualizado na Tabela 1, que apresenta a comparação das médias de QVRS obtidas nas fases pré-TCTH e Enfermaria, a análise dos dados evidenciou diferença estatisticamente significante nos aspectos Capacidade Funcional $(p=0,022)$ e Dor $(p=0,036)$. Esse resultado indica que houve depreciação em tais domí- nios, quando se compararam as fases pré-TCTH e Enfermaria. Entre as demais variáveis da QVRS analisadas pelo SF-36 não foram encontradas diferenças estatisticamente significativas.

Apesar de não terem alcançado significância estatística, as diferenças de escores de qualidade de vida encontradas nas fases pré-TCTH e Enfermaria apontam uma discrepância qualitativamente importante, uma vez que os participantes apresentaram depreciação de diversos domínios da QVRS durante o período de internação (mais precisamente, em seis dos oito domínios avaliados: Aspectos Físicos, Estado Geral de Saúde, Vitalidade, Aspectos Sociais, Aspectos Emocionais e Saúde Mental). Ocorreram pioras na avaliação da Capacidade Funcional, Aspectos Físicos e Aspectos Sociais, bem como nos domínios Dor, Vitalidade e Saúde Mental.

A Tabela 2 mostra a comparação da qualidade de vida, avaliada pelo SF-36, nos momentos pré e pós-TCTH. A análise dos dados não evidenciou diferenças estatisticamente significativas entre as variáveis. Embora as discrepâncias encontradas não sejam estatisticamente significativas, as diferenças de escores de qualidade de vida nas fases pré e pós-TCTH apontam um declínio, na fase pós-TCTH, dos valores de alguns domínios da QVRS: Capacidade Funcional, Aspectos Físicos, Dor e Aspectos Sociais. Já nos aspectos Estado Geral de Saúde e Aspectos Emocionais observou-se melhora na fase pós-TCTH.

A Tabela 3 mostra a comparação da qualidade de vida, avaliada pelo SF-36, nas fases da Enfermaria e pós-TCTH. Na comparação da QVRS entre essas etapas,

Tabela 1. Comparação dos valores médios de qualidade de vida, avaliada pelo SF-36, nas fases pré-TCTH e Enfermaria. Ribeirão Preto (SP), 2009-2010.

\begin{tabular}{|c|c|c|c|c|c|}
\hline \multirow{2}{*}{ Aspectos da QVRS } & \multicolumn{2}{|c|}{ Pré-TCTH } & \multicolumn{2}{|c|}{ Enfermaria } & \multirow{2}{*}{$p$} \\
\hline & M & $\mathrm{DP}$ & M & $\mathrm{DP}$ & \\
\hline Capacidade funcional & 64,3 & 25,0 & 20,0 & 18,5 & $0,022^{*}$ \\
\hline Aspectos físicos & 35,7 & 45,3 & 3,6 & 9,5 & 0,100 \\
\hline Dor & 86,0 & 24,7 & 45,1 & 33,6 & $0,036^{*}$ \\
\hline Estado geral de saúde & 65,5 & 18,2 & 66,1 & 13,5 & 0,675 \\
\hline Vitalidade & 62,5 & 28,7 & 43,6 & 31,5 & 0,151 \\
\hline Aspectos sociais & 69,6 & 36,7 & 21,4 & 21,3 & 0,059 \\
\hline Aspectos emocionais & 28,0 & 48,8 & 33,3 & 47,1 & 1,000 \\
\hline Saúde mental & 72,0 & 29,5 & 68,0 & 14,9 & 1,000 \\
\hline
\end{tabular}

* p 0,05. M: média; DP: desvio-padrão; SF-36: questionário genérico de avaliação de qualidade de vida; TCTH: transplante de células-tronco hematopoéticas; QVRS: qualidade de vida relacionada à saúde. 
Tabela 2. Comparação dos valores médios de qualidade de vida, avaliada pelo SF-36, nas fases pré-TCTH e pós-TCTH. Ribeirão Preto (SP), 2009-2010.

\begin{tabular}{|c|c|c|c|c|c|}
\hline \multirow{2}{*}{ Aspectos da QVRS } & \multicolumn{2}{|c|}{ Pré-TCTH } & \multicolumn{2}{|c|}{ Pós-TCTH } & \multirow{2}{*}{$p$} \\
\hline & M & $\mathrm{DP}$ & M & $\mathrm{DP}$ & \\
\hline Capacidade funcional & 64,3 & 25,0 & 48,5 & 22,6 & 0,151 \\
\hline Aspectos físicos & 35,7 & 45,3 & 7,1 & 18,9 & 0,273 \\
\hline Dor & 86,0 & 24,7 & 61,8 & 24,7 & 0,208 \\
\hline Estado geral de saúde & 65,5 & 18,2 & 78,4 & 10,7 & 0,208 \\
\hline Vitalidade & 62,5 & 28,7 & 59,0 & 24,1 & 0,800 \\
\hline Aspectos sociais & 69,6 & 36,7 & 53,5 & 37,3 & 0,418 \\
\hline Aspectos emocionais & 28,0 & 48,8 & 62,0 & 48,7 & 0,201 \\
\hline Saúde mental & 72,0 & 29,5 & 73,6 & 25,0 & 0,917 \\
\hline
\end{tabular}

* p 0,05. M: média; DP: desvio-padrão; SF-36: questionário genérico de avaliação de qualidade de vida; TCTH: transplante de células-tronco hematopoéticas; QVRS: qualidade de vida relacionada à saúde.

Tabela 3. Comparação dos valores médios de qualidade de vida, avaliada pelo SF-36, nas fases da Enfermaria e pós-TCTH. Ribeirão Preto (SP), 2009-2010.

\begin{tabular}{|c|c|c|c|c|c|}
\hline \multirow{2}{*}{ Aspectos da QVRS } & \multicolumn{2}{|c|}{ Enfermaria } & \multicolumn{2}{|c|}{ Pós-TCTH } & \multirow{2}{*}{$p$} \\
\hline & M & DP & M & $\mathrm{DP}$ & \\
\hline Capacidade funcional & 20,0 & 18,5 & 48,5 & 22,6 & 0,138 \\
\hline Aspectos físicos & 3,6 & 9,5 & 7,1 & 18,9 & 1,000 \\
\hline Dor & 45,1 & 33,6 & 61,8 & 24,7 & 0,402 \\
\hline Estado geral de saúde & 66,1 & 13,5 & 78,4 & 10,7 & $0,036^{*}$ \\
\hline Vitalidade & 43,6 & 31,5 & 59,0 & 24,1 & 0,075 \\
\hline Aspectos sociais & 21,4 & 21,3 & 53,5 & 37,3 & 0,093 \\
\hline Aspectos emocionais & 33,3 & 47,1 & 62,0 & 48,7 & 1,000 \\
\hline Saúde mental & 68,0 & 14,9 & 73,6 & 25,0 & 0,402 \\
\hline
\end{tabular}

* $p \leq 0,05$. M: média; DP: desvio-padrão; SF-36: questionário genérico de avaliação de qualidade de vida; TCTH: transplante de células-tronco hematopoéticas; QVRS: qualidade de vida relacionada à saúde.

a análise dos dados evidenciou diferença estatisticamente significativa no aspecto Estado Geral de Saúde $(p=0,036)$, que aparece mais rebaixado na Enfermaria. Entre as demais variáveis não foram encontradas diferenças estatisticamente significativas. As diferenças obtidas entre as médias dos escores sugerem uma avaliação inferior dos aspectos da qualidade de vida na fase da Enfermaria.

\section{Discussão}

A literatura ressalta o impacto que as doenças neoplásicas provocam nos pacientes e em seus familiares (Deitos \& Gaspary, 1997; Peres \& Santos, 2006a; Valle, 1994). A descoberta de um câncer hematológico é um possível desencadeador de transtornos de ajusta-

192 mento nos pacientes (Peres \& Santos, 2005; 2006b), daía necessidade de avaliação da qualidade de vida com vistas a melhorar as indicações terapêuticas para cada caso. Assim, é de fundamental importância que a equipe multidisciplinar de saúde, responsável pela condução do tratamento, compreenda a dinâmica envolvida na relação paciente-família-cuidado e identifique a influência que os fatores psicossociais exercem nesse contexto, facilitando, assim, o processo de adaptação à nova realidade.

Após o diagnóstico, um novo confronto altamente impactante pode advir, com a tomada de decisão pela realização ou não do tratamento. O paciente passa pelas fases de seleção de possíveis doadores, preparo para o transplante, o transplante propriamente dito, possíveis complicações decorrentes da elevada toxicidade da quimioterapia e dos procedimentos invasivos, a tão aguardada "pega da medula" e a alta da unidade 
hospitalar para acompanhamento ambulatorial (Oliveira-Cardoso et al., 2009b; Peres \& Santos, 2006c). Considerando que essas etapas acentuam o estresse e potencializam reações de ansiedade e depressão, a equipe de saúde deve estar preparada para oferecer um ambiente de confiança e acolhimento às necessidades apresentadas pelos pacientes e seus cuidadores. Uma comunicação clara e baseada em vocabulário simples deve prevalecer no contato entre profissionais, pacientes e familiares, de modo a permitir a assimilação de inúmeras informações, com diferentes níveis de complexidade. Também se devem respeitar as expectativas, crenças e valores de cada indivíduo e de seu contexto sociofamiliar. Essas atitudes podem ajudar a minimizar as consequências negativas que um procedimento tão radical e agressivo como o TCTH acarreta para a qualidade de vida dos pacientes (Matsubara et al., 2007).

Corroborando o que já está claramente documentado na literatura (Oliveira-Cardoso, Santos, Mastropietro \& Voltarelli, 2010; Peres \& Santos, 2006b), o transplante surge para esses pacientes como uma possibilidade resolutiva, uma vez que a recuperação da saúde e dos aspectos a ela relacionados dependem dessa opção terapêutica. Os pacientes tendem a se agarrar na possibilidade dessa intervenção dar certo, por mais que os riscos e fatores adversos associados ao procedimento sejam enfatizados pela equipe no momento de se ponderar a relação custo-benefício, durante o delicado processo de conduzir a tomada de decisão. Assim, os indivíduos que têm indicação para se submeterem ao TCTH tendem a se fixar nos possíveis benefícios que a terapêutica pode lhes trazer, nutrindo expectativa de recuperação substancial da saúde e dos aspectos vitais relacionados à qualidade de vida que foram prejudicados pela doença.

Apesar dessa possibilidade ser cada vez mais plausível, na medida em que o avanço do conhecimento na área permite a obtenção de resultados mais seguros e duradouros, o transplante ainda é vivenciado como uma experiência nova e ameaçadora, pois tanto pode salvar, como levar ao óbito (Álvares et al., 2003; Oliveira-Cardoso et al., 2010), o que interfere significativamente na qualidade de vida dos pacientes. Dessa maneira, a decisão pela realização do TCTH geralmente vem acompanhada pela esperança de cura, mas também pode ser desencadeadora de sentimentos ambivalentes, de esperança e medo do que possa acontecer no futuro. Esse incremento de incertezas potencializa conflitos psicológicos mobilizadores de ansiedade, devido à complexidade e aos riscos potenciais envolvidos. Esses fatores adversos contribuem para depreciar a qualidade de vida.

Os dados obtidos no presente estudo sugerem a potencial vulnerabilidade psicológica dos pacientes nas vésperas da realização do transplante. Assim, o rebaixamento de aspectos avaliados pelo SF-36, como Estado Geral de Saúde, Aspectos Físicos e Emocionais, pode guardar relação com a fragilidade física decorrente do agravamento da doença de base e também com a ambivalência de sentimentos despertados com a tomada de decisão pela realização do transplante. A situação paradoxal de risco e esperança suscita nos participantes emoções extremas e ambivalentes, como euforia e choque, otimismo e temor de que o procedimento não dê certo. O medo de morrer pode ser recalcado na busca de uma postura resignada frente ao que não se pode modificar, de conformismo diante da oportunidade representada pelo novo tratamento, da busca de conforto e serenidade, e até mesmo na felicidade por ter, finalmente, chegado a hora de poder se submeter a uma terapêutica que parece promissora, como ocorre no contexto de outros tipos de transplante (Ravagnani, Domingos \& Miyazaki, 2007).

A qualidade de vida do paciente no período prévio à realização do transplante pode interferir no modo como ele vivencia a passagem pelo isolamento protetor da Enfermaria e as mudanças radicais que ocorrem em seu corpo. O modo como o paciente irá reestruturar sua vida, após a alta hospitalar e o retorno ao lar, pode estar associado com a maneira como ele vivenciou as etapas anteriores do tratamento. Nessa vertente, destaca-se a importância de uma exploração minuciosa da situação social e psicológica do paciente candidato ao TCTH (Peres \& Santos, 2006b; 2006c), visto que muitos sofrem com questões relacionadas a uma incipiente preparação psicológica e social. A rede de apoio social também precisa ser apreciada. Assim, Krasuska, Dmoszynska, Daniluke Stanislawek (2002) apontam para a importância de desenvolver recursos educativos, como disponibilizar uma cartilha de informações ao paciente, elaborada em linguagem simples e clara, com o objetivo de esclarecer dúvidas e responder às perguntas iniciais. 
Durante oTCTH propriamente dito, os pacientes permanecem confinados em um quarto, em regime de total isolamento protetor, enquanto aguardam a infusão da nova medula e recebem a quimioterapia que induz a imunossupressão, o que aumenta exponencialmente o risco de contrair infecções oportunistas. Por passarem um longo período nesse estado de confinamento e sistema imunológico aniquilado, não é raro que desenvolvam reações de ajustamento à situação adversa, permeada por ansiedade, irritabilidade e tédio. Em casos extremos, podem apresentar grave descompensação psíquica, inclusive com vivências de despersonalização. Adiciona-se a esse quadro preocupante a constante ameaça de ocorrerem complicações decorrentes do próprio procedimento do transplante, como a temida Doença do Enxerto Contra o Hospedeiro (DECH), que pode potencializar a exposição a fatores estressores e aumentar o risco de morte (Álvares et al., 2003; Peres \& Santos, 2006b).

Pontes, Guirardello e Campos (2007) apontam, com base em um estudo de caso, que o paciente que vivencia essa situação sofre com a ausência da família, o medo da morte e a incerteza quanto ao futuro, além de experimentar sentimentos de compaixão, proteção exagerada e imprecisão de informações, mesclados com o excesso de manuseio pela equipe de enfermagem. Deve-se acrescentar ainda a desorientação causada pela ruptura da vida cotidiana, com o afastamento do trabalho e a restrição de espaço físico. O incômodo sentido com o isolamento social e o afastamento da família é agravado pela imobilidade física e pela limitação a um ambiente hospitalar assético e monótono. A essas restrições são adicionados os efeitos colaterais dos quimioterápicos, a ocorrência de mudanças profundas na autoimagem e a necessidade de manterem um acompanhante dia e noite, o que reforça a perda de autonomia e o estado de regressão e dependência extrema em que se encontram no período de enfermaria. Todas essas alterações, que correspondem às possíveis implicações físicas e psicossociais do procedimento, podem contribuir para o rebaixamento da qualidade de vida (Guimarães et al., 2010). Com efeito, os dados obtidos na presente investigação corroboram que a QVRS dos pacientes nessa etapa do tratamento encontra-se em sua pior fase.

Apesar dos piores índices de qualidade de vida 194 terem sido obtidos na etapa da Enfermaria, observa-se que, comparados com os índices do pré-TCTH, os domínios relativos ao Estado Geral de Saúde e Aspectos Emocionais encontravam-se melhor preservados durante a internação. Isso pode estar relacionado com o acolhimento e o suporte recebidos da equipe de saúde, que inclui profissionais de saúde mental, como psicólogo, psiquiatra e terapeuta ocupacional (Oliveira, Santos \& Mastropietro, 2005). Esses profissionais oferecem apoio aos pacientes durante o período de internação e a assistência é continuada nos retornos ambulatoriais após a alta hospitalar. Um plano de intervenção é traçado, buscando-se fortalecer as estratégias de enfrentamento que promovem adaptação a cada etapa vivenciada nessa longa jornada (Oliveira \& Santos, 2009).

Nesse sentido, Miyazaki, Domingos, Valério, Santos e Rosa (2002) descrevem a importância do serviço de Psicologia e da equipe de saúde mental nas enfermarias. Destacam que, nas enfermarias, as intervenções realizadas pela equipe de saúde mental têm por objetivo facilitar o vínculo com a equipe interdisciplinar, de modo a minimizar as dúvidas e crenças incorretas acerca da doença e do tratamento, favorecendo o desenvolvimento de recursos adaptativos frente às restrições impostas.

Os resultados obtidos devem ser vistos com a devida cautela, dado o número reduzido de participantes do estudo e a grande variabilidade das medidas obtidas. Nesse sentido, chama a atenção, por exemplo, o fato de que, no pré-TCTH, em um domínio como Aspectos Emocionais, cinco participantes obtiveram pontuação nula, enquanto os outros dois alcançaram pontuação máxima (100).

\section{Considerações Finais}

Atualmente, oTCTH é utilizado para o tratamento de diversas doenças neoplásicas - como leucemias, linfomas, mieloma múltiplo - e não neoplásicas - a exemplo da aplasia medular, hemoglobinopatias, distúrbios metabólicos ou imunológicos congênitos e doenças autoimunes (Mendes et al., 2010). Esse amplo espectro de indicações evidencia a necessidade de investir em pesquisas que considerem o potencial impacto para a qualidade de vida dos pacientes, como mostra o presente estudo. 
Com a alta hospitalar foi observada melhora na qualidade de vida dos pacientes, em comparação com a etapa da Enfermaria. Mesmo que discreta, a melhora constatada nas dimensões analisadas pode ter um impacto importante e positivo sobre o bem-estar psicológico e a funcionalidade diária do paciente (Ravagnani et al., 2007).

Ao se comparar a qualidade de vida durante o pré e o pós-TCTH, observou-se que os indicadores foram superiores no pré-TCTH. Após a realização do transplante, a QVRS dos pacientes sofreu o impacto das implicações decorrentes do procedimento. Eles enfrentaram limitações físicas, dores, sensação de distorção da autoimagem, efeitos colaterais do tratamento, que se somaram às alterações duradouras nos hábitos de vida - como a perda ou prejuízo da capacidade produtiva, da autonomia, da função sexual e da fertilidade, além dos papéis sociais (Lesko, 1990). Não obstante tais prejuízos, os domínios Estado Geral de Saúde e Aspectos Emocionais encontravam-se melhorados no pós-TCTH, provavelmente devido aos cuidados intensivos recebidos da equipe de saúde e ao suporte psicoterápico e em terapia ocupacional regularmente oferecidos (Oliveira-Cardoso, Santos, Mastropietro, Sponholz Jr \& Voltarelli, 2009a).

Pelo exposto, nota-se que, no pós-TCTH imediato, apesar do alívio advindo da saída da enfermaria, a qualidade de vida continuou prejudicada em diversos aspectos. Por outro lado, durante os 100 dias que se sucederam à infusão da nova medula óssea, a melhora na qualidade de vida, mesmo que discreta, pode representar um impacto positivo sobre o bem-estar e a funcionalidade diária do paciente, considerando a acentuada situação de vulnerabilidade em que ele se encontra. Tal melhora foi sentida e relatada pelos pacientes que, gradualmente, começaram a expressar o desejo de retomar suas atividades, dedicar-se mais à família, ter mais tempo para si mesmos, bem como ressignificar o viver após a realização do transplante.

Com a alta hospitalar, o paciente começa a vislumbrar a retomada de suas atividades rotineiras e/ou o recomeço de sua vida. Porém, vale ressaltar que não se pode esperar que retome de uma só vez tudo o que interrompera. É preciso respeitar o tempo necessário para a retomada de sua autonomia e da linha de continuidade do seu existir.
Com a evolução e aprimoramento progressivo da técnica do TCTH, muitas melhoras vêm sendo incrementadas, o que permite elevar a expectativa de sobrevida dos pacientes com indicação para esse tipo de procedimento. Desse modo, a qualidade de vida do paciente passa a ser tão relevante quanto sua sobrevida, o que torna necessário empreender melhorias nas modalidades de assistência integral ao transplantado e sua família em todas as etapas do prolongado percurso de tratamento. Também é necessária uma maior atenção à formação e qualificação de profissionais envolvidos no atendimento a essa população.

É preciso mencionar os limites do presente estudo, como a casuística reduzida e a aplicação de apenas um instrumento genérico de avaliação da QVRS. Sugere-se a necessidade de estudos futuros, com uso combinado do SF-36 com um instrumento específico de qualidade de vida, como a escala FACT-BMT.

Finalmente, com base nos resultados analisados, pode-se concluir que melhorar a qualidade de vida e a adaptação dos pacientes à realidade do TCTH deve se constituir em um importante foco de intervenção e assistência no decurso do tratamento. Considera-se que essa deveria ser uma preocupação imperiosa da equipe responsável pelo transplante a curto, médio e longo prazo, ao lado da atenção dada à recuperação hematopoética pós-transplante. Além disso, são necessários novos estudos, com uma casuística ampliada e delineamentos mais sofisticados de pesquisa, para assegurar o enriquecimento do conhecimento produzido nessa área e garantir melhores condições de assistência e acompanhamento dos pacientes submetidos ao TCTH.

\section{Referências}

Álvares, M., Bach, C., \& Campos, E. M. P. (2003). Estados emocionais do paciente candidato a transplante de medula óssea. Psicologia: Teoria e Prática, 5 (2), 23-36.

Ciconelli, R. M. (1997). Tradução para o português e validação do Questionário Genérico de Avaliação de Qualidade de Vida Medical Outcomes Study 36 -Item Short-form Health Survey (SF-36). Tese de doutorado não-publicada, Universidade Federal de São Paulo.

Cooper, M. C., \& Powell, G. (1998). Technology and care in bone marrow transplantation unit: creating and assuaging vulnerability. Holistic Nursing Practice, 12 (4), 57-68.

Deitos, T.F. H., \& Gaspary, J. F.P. (1997). Efeitos biopsicossociais e psiconeuroimunológicos do câncer sobre pacientes e familiares. Revista Brasileira de Cancerologia, 43 (2), 117-126. 
Fife, B. L., Huster, G. A., Cornetta, K. G., Kennedy, V. N., Akard, L.P., \& Broun, E. R. (2000). Longitudinal study of adaptation to the stress of bone marrow transplantation. Journal of Clinical Oncology, 18 (7), 1539-1549.

Fleck, M. P., Leal, O. F., Louzada, S., Xavier, M., Cachamovich, E., Vieira, G., et al. (1999). Desenvolvimento da versão em português do instrumento de avaliação de qualidade de vida da OMS (WHOQoL-100). Revista Brasileira de Psiquiatria, 21 (1), 21-28.

Goetzmann, L., Klaghofer, R., Wagner-Huber, R., Halter, J., Boehler, A., Muellhaupt, B., et al. (2007). Psychosocial vulnerability predicts psychosocial outcome after an organ transplant: results of a prospective study with lung, liver, and bone-marrow patients. Journal of Psychosomatic Research, 62 (1), 93-100.

Gregurek, R., Brajkovic, L., Kalenic, B., Bras, M., \& Persic-Brida, M. (2009). Five years study on impact of anxiety on quality of life in patients treated with bone marrow transplantation. Psychiatric Danub, 21 (1), 49-55.

Guimarães, F. A. B., Santos, M. A., \& Oliveira, E. A. (2008). Quality of life of patients with autoimmune diseases submitted to bone marrow transplantation: a longitudinal study. Revista Latino-Americana de Enfermagem, 16 (5), 856-863.

Guimarães, F. A. B., Oliveira-Cardoso, E. A. O., Mastropietro, A. P., Voltarelli, J. C., \& Santos, M. A. (2010). Impact of autologous hematopoeitic stem cell transplantation on the quality of life of patients with multiple sclerosis. Arquivos de Neuro-Psiquiatria, 68 (4), 522-527.

Haberman, M., Bush, N., Young, K., \& Sullivan, K. M. (1993). Quality of life of adult long-term survivors of bone marrow transplantation: a quality analysis of narrative data. Oncology Nursing Forum, 20 (10), 1545-1553.

Hann, D. M., Jacobsen, P. B., Martinho, S. C., Kronish, L. E., Azzarello, L. M., \& Fields, K.K. (1997). Quality of life following bone marrow transplantation for breast comparative study. Bone Marrow Transplantation, 19 (3), 257-264.

Heinonen, H., Volin, L., Zevon, M. A., Uutela, A., Barrick, C., \& Ruutu, T. (2005). Stress among allogeneic bone marrow transplantation patients. Patient Education and Counseling, $56(1), 62-67$.

Krasuska, M. E., Dmoszynska, A., Daniluk, J., \& Stanislawek, A. (2002). Information needs of the patients undergoing bone marrow transplantation. Annales Universitatis Mariae Curie Sklodowaska, Sectio D: Medicina, 57 (2), 178-185.

Lesko, L. M. (1990). Bone marrow transplantation. In J. Holland \& J. Rowland (Eds.), Handbook of psychooncology: psychological care for the patient with cancer (pp.163-172). New York: Oxford University Press.

Mastropietro, A. P., Oliveira-Cardoso, E. A., Simões, B. P., Voltarelli, J. C., \& Santos, M. A. (2010). Relação entre renda, trabalho e qualidade de vida de pacientes submetidos ao transplante de medula óssea. Revista Brasileira de Hematolologia e Hemoterapia, 32 (2), 102-107.

Matsubara, T. C., Carvalho, E. C., Canini, S. R. M. S., \& Sawada, N. O. (2007). A crise familiar no contexto do transplante de medula óssea (TMO): uma revisão integrativa. Revista Latino-Americana de Enfermagem, 15 (4), 665-670.
McQuellon, R. P., Russell, G., Rambo, T. D., Craven, B. L., Radford, J., Perry, J. J., Cruz, J., et al. (1998). Quality of life and psychological distress of bone marrow transplant recipients: the time trajectory's recovery over the first year. Bone Marrow Transplantation, 21 (5), 477-486.

Mendes, M. L., Bittencourt, H. N. S., \& Dusse, L. M. S. (2010). Fontes de células no transplante de células-tronco hematopoéticas. Revista Brasileira de Análises Clínicas, 42 (1), 3-7.

Miyazaki, M. C. O. S., Domingos, N. A. M., Valério, N. I., Santos, A. R., \& Rosa, L. T. B. (2002). Psicologia da saúde: extensão de serviços à comunidade, ensino e pesquisa. Psicologia USP, 13 (1), 29-53.

Molassiotis, A., \& Morris, P. J. (1999). Quality of life in patients with chronic myeloid leukemia after unrelated donor bone marrow transplantation. Cancer Nursing, 22 (5), 340-349.

Oliveira, E. A., \& Santos, M. A. (2009). Qualidade de vida de pacientes submetidos ao transplante de medula óssea. In S. R. Pasian, G. Romanelli \& M. V. Cunha (Orgs.), Investigação científica em psicologia: aplicações atuais em saúde (pp.155-182). São Paulo: Vetor.

Oliveira, E. A., Santos, M. A., \& Mastropietro, A. P. (2005). Oficinas terapêuticas: uma possibilidade de intervenção em saúde mental. In C. P. Simon, L. L. Melo-Silva \& M. A. Santos (Orgs.), Formação em psicologia: desafios da diversidade na pesquisa e na prática (pp.369-378). São Paulo: Vetor.

Oliveira, E. A., Santos, M. A., Mastropietro, A. P., \& Voltarelli, J. C. (2009). Qualidade de vida pós-TMO. In L. N. Machado, V. O. Camandoni, K. P. H. Leal \& E. L. M. Mascatello (Orgs.), Transplante de medula óssea: abordagem multidisciplinar (pp.159-174). São Paulo: Lemar.

Oliveira-Cardoso, E. A., Santos, M. A., Mastropietro, A. P. Sponholz Jr, A., \& Voltarelli, J. C. (2009a). Intervenções da psicologia, psiquiatria e terapia ocupacional no transplante de células-tronco hematopoéticas. In J. C. Voltarelli, R. Pasquini \& E. T. T. Ortega (Orgs.), Transplante de células-tronco hematopoéticas (pp.1099-1118). São Paulo: Atheneu.

Oliveira-Cardoso, E. A., Santos, M. A., Mastropietro, A. P., \& Voltarelli, J. C. (2009b). Qualidade de vida de sobreviventes do transplante de medula óssea (TMO): um estudo prospectivo. Psicologia: Teoria e Pesquisa, 25 (4), 529-536.

Oliveira-Cardoso, E. A., Santos, M. A., Mastropietro, A. P., \& Voltarelli, J. C. (2010). Bone marrow donation from the perspective of sibling donors. Revista Latino-Americana de Enfermagem, 18 (5), 911-918.

Orley, J., \& Kuyken, W. (1994). Quality of life assessment: international perspectives. Berlin: Springer-Verlag.

Peres, R. S., \& Santos, M. A. (2005). Carência fantasmática e vulnerabilidade somática em pacientes onco-hematológicos com indicação para o transplante de medula óssea. Psicologia Hospitalar, 3 (1), 85-101.

Peres, R. S., \& Santos, M. A. (2006a). A exclusão do afeto e a alienação do corpo. São Paulo: Vetor.

Peres, R. S., \& Santos, M. A. (2006b). Relações entre a personalidade dos pacientes e a sobrevivência após o trans- 
plante de medula óssea: revisão da literatura. Psicologia em Estudo, 11 (2), 341-349.

Peres, R. S., \& Santos, M. A. (2006c). Contribuições do Inventário Fatorial de Personalidade (IFP) para a avaliação psicológica de pacientes onco-hematológicos com indicação para o transplante de medula óssea. Psicologia em Revista, 12 (19), 22-33.

Peres, R. S., \& Santos, M. A. (2009). Aspectos psicossomáticos do transplante de medula óssea: uma leitura psicanalítica pós-freudiana. In S. R. Pasian, G. Romanelli \& M. V. Cunha (Orgs.), Investigação científica em psicologia: aplicações atuais em saúde (pp.139-154). São Paulo: Vetor.

Pontes, L., Guirardello, E. B., \& Campos, C. J. G. (2007). Demandas de atenção de um paciente na unidade de transplante de medula óssea. Revista da Escola de Enfermagem da USP, 41 (1), 154-160.

Ravagnani, L. M. B., Domingos, N. A. M., \& Miyazaki, M. C. O. S. (2007). Qualidade de vida e estratégias de enfrentamento em pacientes submetidos a transplante renal. Estudos de Psicologia (Natal), 12 (2), 177-184.
Riul, S., \& Aguillar, O. M. (1997). Contribuição à organização de serviços de transplante de medula óssea e a atuação do enfermeiro. Revista Latino-Americana de Enfermagem, 5 (1), 49-58.

Thomas, E. D. (2000). Bone marrow transplantation: a historical review. Medicina, 33 (3), 209-218.

Trask, P. C., Paterson, A., Riba, M., Brines, B., Griffith, K., Parker, P., et al. (2002). Assessment of psychological distress in prospective bone marrow transplant patients. Bone Marrow Transplantation, 29 (11), 917-925.

Valle, E. R. M. (1994). Algumas conseqüências psicossociais em crianças curadas de câncer: visão dos pais. Jornal de Pediatria, 70 (1), 21-27.

Ware, J. E. Jr., \& Sherbourne, C. D. (1992). The MOS 36-Item Short-Form Health Survey (SF-36): I. Conceptual framework and item selection. Medical Care, 30 (6), 473-483.

Recebido em: 23/3/2010

Aprovado em: 11/3/2011 
